(Aus der Neurologischen Klinik [Prof. Dr. Carl D. Camp] und der Psychiatrischen Klinik [Prof. Dr. Albert M. Barrett] der Universität Michigan zu Ann Arbor [Michigan USA.].)

\title{
Konzentrische Sklerose.
}

\author{
Von \\ R. Waggoner und K. Löwenberg.
}

Mit 6 Textabbildungen.

(Eingegangen am 26. August 1933.)

Unter denjenigen Erkrankungen des Z. N. S., welche mit ausgedehnten Entmarkungen einhergehen und zu den verschiedenen Untergruppen der diffusen Sklerose gerechnet werden, ist die von Baló beschriebene konzentrische Sklerose eine der auffallendsten. Die Zahl der Beobachtungen, welche hierher gehören, ist eine sehr geringe, sie stammen von Marburg, Barré, Hallervorden-Spatz und Patrassi.

Eine weitere Beobachtung soll hier besprochen werden.

Klinischer Bericht. L. W., 17 Jahre alt, Schüler, wurde am 28.10.1931 in die neurologische Klinik aufgenommen. Er klagte über Doppeltsehen und Störungen beim Sprechen, Schlucken und Gehen. Der Kranke gab an, daß er etwa 2 Monate vor seiner Errkrankung an allgemeiner Schwäche und Mattigkeit gelitten habe und. gezwungen war, den Schulbesuch zeitweise zu unterbrechen.

7 Wochen vor der Aufnahme glitt er beim Baden vom Sprungbrett ab und schlug mit der linken Kopfseite auf das Wasser. Er verspürte heftige Kopfschmerzen, verlor aber das Bewußtsein nicht. Obwohl er schwindlig wurde und beim Gehen stolperte, konnte er zu Fuß nach Hause gehen. Der Kranke erbrach später und, konnte nichts zu sich nehmen, erholte sich aber soweit, daß er imstande war Fußball zu spielen und besuchte am gleichen Abend die Lichtspiele. Am nächsten Morgen klagte er über Doppeltsehen und Schwindel. Der Arzt verordnete Bettruhe und der Kranke verblieb im Bett bis zur Aufnahme in die Klinik. In den letzten 3 Wochen vor der Aufnahme wurde die Sprache zusehends schlechter und es traten profuse Schweiße auf, sowie Gesichtskrämpfe und Schluckstörungen. Die Familiengeschichte war ohne Belang. Der Kranke hatte als Kind Masern, Mumps und Keuchhusten durchgemacht, seine Gesundheit war sonst gut. Er war ein durchschnittlicher Schüler.

Die körperliche Untersuchung ergab einen jungen Mann von normalem Körperbau und gutem Ernährungszustand. Herz und Lungen waren gesund. Die abdominale Muskulatur war gespannt, aber das Abdomen war nicht druckempfindlich.

Der Kranke war gut orientiert und beantwortete die an ihn gestellten Fragen richtig. Die Augen konnten nicht nach rechts gerichtet werden, wohl aber nach oben und unten. Beim Blick nach oben trat ein langsamer rhythmischer Nystagmus 
auf. Das rechte Oberlid war gesenkt, nach der Angabe des Kranken um Doppeltsehen auszuschalten. Die rechte Pupille war etwas größer als die linke, beide reagierten auf Licht prompt, auf Akkommodation träge. Beiderseits bestand eine Neuroretinitis. Der linke Facialis war fast völlig gelähmt; die Zunge wurde gerade und ohne Tremor ausgestreckt. Biceps- und Tricepsreflexe waren beiderseits normal; beide Arme waren etwas ataktisch.

Die Abdominalreflexe konnten nicht ausgelöst werden, da die Bauchmuskeln gespannt waren. Knie- und Achillesreflexe waren normal, Babinski und Kernig waren nicht nachweisbar, es bestand keine Nackenstarre.

Die Sprache war von bulbärem Typus; Mimik und Lidschlag waren verlangsamt. Die Hörfähigkeit war links etwas herabgesetzt, aber genauere Bestimmungen gelangen nicht, da der Kranke den Aufforderungen nicht nachkommen konnte.

Röntgenaufnahmen des Schädels und der inneren Organe ergaben nichts Krankhaftes.

Der Liquordruck betrug $150 \mathrm{~mm}$ bei normaler Zellzahl, Kahn-, Mastix-, Goldsohl- und Globulinreaktionen waren negativ, Kultur steril, Inokulation eines Meerschweinchens mit dem Liquor verlief resultatlos. Der Liquor enthielt $75 \mathrm{mg}$ Zucker pro $100 \mathrm{~cm}$, der Blutzucker betrug $103 \mathrm{mg}$ pro $100 \mathrm{ccm}$. Blutkultur negativ, Kahn im Blut negativ.

Blutbild: Hgl. $80 \%$, weiße 6950 , davon Poly. $72 \%$, basoph. $3 \%$, eosin. $4 \%$, mono. $2 \%$, große Lymphkörperchen $18 \%$, rote 5020000 .

5 Tage nach der Aufnahme war der Befund im wesentlichen unverändert, abgesehen von einer beträchtlichen Rötung des rechten Auges und der rechten Gesichtshälfte; das Auge war etwas lichtscheu und die Tränensekretion war etwas verstärkt. 3 Tage später bestand ein beträchtlicher Tränenfluß beiderseits. Die Beweglichkeit des rechten Auges war nunmehr normal, das linke war jedoch gelähmt. Zu gleicher Zeit machten sich Zeichen einer Blasenlähmung bemerkbar. Die Zunge wich nach links $a b$ und der Speichelfluß war verstärkt. Knie- und Achillessehnenreflexe waren beiderseits lebhaft; links trat Babinski auf. Am 30. 10. verlor die Sprache ihren bulbären Charakter und wurde explosiv, das Hörvermögen erlosch beiderseits fast plötzlich. Die Sprache besserte sich in den nächsten Tagen, aber das Gehör kehrte nicht mehr zurück. Das Sensorium war bereits merklich getrübt. Am 9. 11. wurde eine weitere Versehlimmerung verzeichnet: Das Schlucken wurde sehr erschwert, die Sprache wurde ganz undeutlich und dysarthrisch, es bestand eine fast komplette Ophthalmoplegia externa. Im Laufe des Tages hörte spontanes Sprechen auf, beide Gesichtshälften waren fast völlig gelähmt, rechts mehr als links. Die Zunge konnte bewegt werden, aber die Zungenränder zuckten. Die Sehnenreflexe blieben im wesentlichen unverändert. Babinski, Oppenheim, Schaffer waren rechts positiv. 2 Tage später war das Schlucken unmöglich und der Kranke mußte mit der Sonde gefüttert werden; der Darm war träge. Nachts trat starke Unruhe auf, der Kranke schien blind zu sein, eine Verständigung war mit ihm nicht mehr möglich. In diesem Zustand verblieb er bis zum 21. 11., dann traten Symptome einer Bronchopneumonie hinzu und der Tod erfolgte 3 Tage später.

Bis zum Auftreten der Pneumonie blieb die Temperatur normal, der Puls schwankte zwischen 60 und 100, die Atmung betrug im Durchschnitt 20 pro Minute, eine Erhöhung auf 45-50 erfolgte in den letzten Todesstunden.

Zusammenfassung: Junger Mann von 17 Jahren, erkrankte nach länger anhaltenden unbestimmten Prodromalsymptomen im Anschluß an einen leichten Unfall; es stellten sich Schwindel, Erbrechen, Doppeltsehen und Schwierigkeiten beim Gehen ein. Im Laufe von 3 Wochen traten Schluck und Sprachbeschwerden, Gesichtskrämpfe, Schweißausbrüche und Schluckstörungen hinzu.

Bei der Aufnahme war das Rotieren der Augen nach rechts und links unmöglich, es bestand ein langsamer vertikaler Nystagmus, das rechte Augenlid war gesenkt, 
die rechte Pupille war größer als die linke und beide reagierten träge. Es bestand beiderseits eine Neuroretinitis. Der linke Facialis war fast völlig gelähmt, das Gehör war herabgesetzt. Die Reflexe waren zunächst ohne gröbere Abweichungen und pathologische bestanden nicht. Blut und Liquor waren normal.

Der Zustand des Kranken verschlimmerte sich schnell, Sprache, Gehör und Schluckvermögen gingen verloren, alle Extremitäten waren sub finem gelähmt, es bestand eine fast komplette Ophthalmoplegia externa. Babinski, Oppenheim und Schaffer traten rechts auf. Der Verlauf war fieberfrei bis zum Auftreten einer terminalen Pneumonie. Die Krankheitsdauer betrug etwa 12 Wochen.

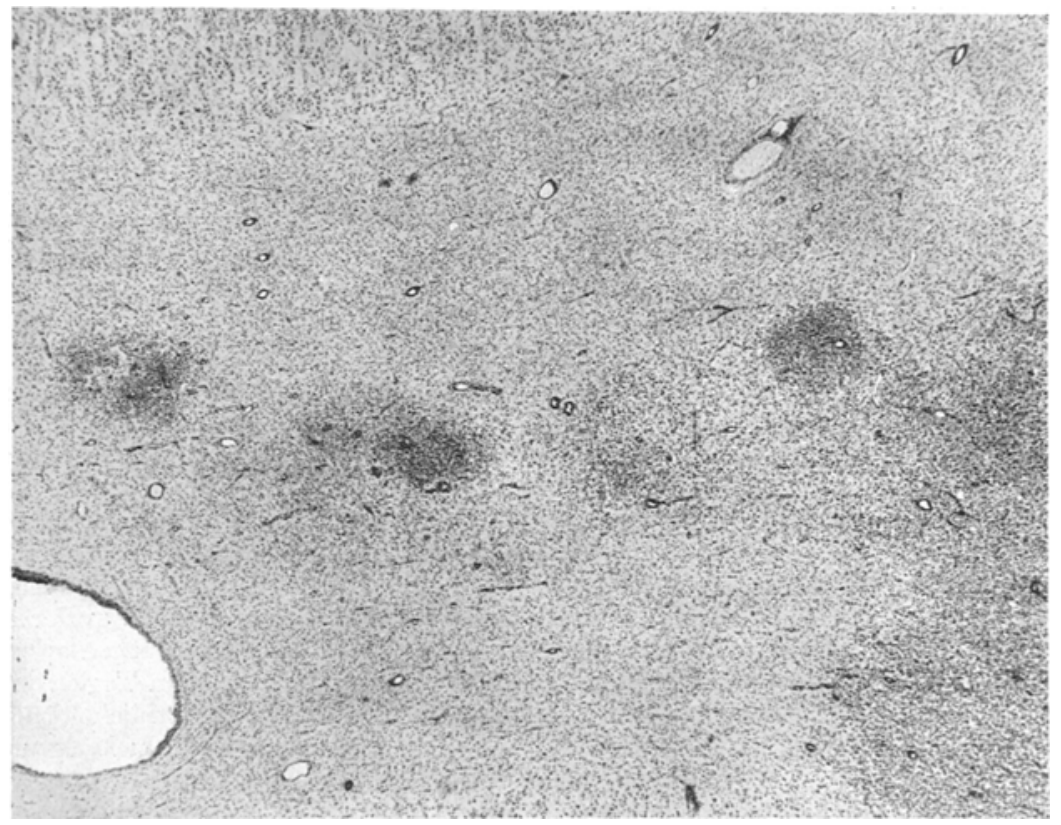

Abb. 1. Kleine perivasculäre Herde im Occipitalmark: Gefäße ron Lymphocyten umgeben. Rechts im Bilde ein gröBerer Herd. Leichte diffuse Reizung des Markes. Nissl-Färbung; $10 \mathrm{mal}$. Mikrophpotogramm.

Sektionsbefund. Die Leptomeningen waren etwas injiziert, sonst aber obne Besonderheiten. Die basalen Gefäße waren zart, die Windungen normal entwickelt. Auf Horizontalschnitten war das Rindengrau von normaler Breite und Aussehen. Das Marklager erschien im Bereiche des linken Parietal-, Temporal- und Occipitallappens graublärlich gefärbt und sehr weich, zum Teil erschien es mehr graurosa. Die Störung griff auch auf die Stammganglien der linken Seite über und zerstörte einen beträchtlichen Teil der inneren Kapsel, des linken Thalamus sowie des Pallidum und Putamen. Gleiches Bild bot der Gehirnstamm, die Brücke und der Boden des 4. Ventrikels. Das Kleinhirn war normal. Die Körpersektion ergab nichts Pathologisches bis auf eine hypostatische Pneumonie.

Histologischer Befund. Die jüngsten Stadien der Markerkrankung wurden im Temporal- und Occipitalgebiet beobachtet. Hier bildeten sich kleine perivasculäre Herde, welche im Nissl-Schnitt als blaue, mit dem unbewaffneten Auge noch eben sichtbare Punkte, erkennbar waren. Dieselben bestanden aus Gitterzellen, Hortegaelementen und Astrocyten; ein stets im Herde gelegenes Gefäß war meistens von 
Lymphocyten umgeben (Abb. 1). Diese Herde waren relativ gut umrissen, obwohl das umgebende Mark etwas gereizt erschien und zerstreute Astrocyten und perivasculäre Lymphocyten enthielt.

An den Gefäßen im Bereiche der Herde waren nur unbedeutende Veränderungen sichtbar, Schwellungen und. Schrumpfungen des Endothels, sowie leichte Reizung der Adventitia, aber keine Nekrosen. Die perivasculären Herde erschienen an zahlreichen Stellen des Markes gleichzeitig und flossen, sich wie Ölflecke ausbreitend, zusammen, wodurch immer größere Abschnitte der weißen Substanz eingeschmolzen wurden (Abb. 2). Durch fortwährend neu hinzutretende Herde kam es an der Peripherie der befallenen Areale zur Bildung eines sehr zellreichen Walles. Diese Wallbildung war sehr deutlich, da in den zentralen, also älteren Teilen der Herde der Zellreichtum ein geringerer und die Zellverteilung eine gleichmäßigere war als in den jüngeren, peripheren (Abb. 2 und 3).

Das histologische Bild war in den Stammganglien, den subthalamischen Zentren, Pons und Medulla im wesentlichen ein gleiches, nur war in all diesen Gebieten das Parenchym beträchtlich in Mitleidenschaft gezogen.

Hin und wieder griff die Erkrankung vom Mark aus auf beschränkte Abschnitte der Rinde über. Diese Herde enthielten hauptsächlich schön ausgebildete fett. beladene Hortegaelemente.

Scharlachrote Schnitte ergaben das bekannte Bild des Lypoidabbaues mit zahllosen fettbeladenen Körnchenzellen, frei im Gewebe oder in den perivasculären Räumen gelegen. Die Fettpräparate ergaben außerdem eine Reihe interessanter Einzelheiten. An der Peripherie der befallenen Areale ließen sich 3 Zonen unterscheiden: eine äußere ganz schmale, welche sich mit Sudan und Scharlach schwach färbte und in deren Bereich die Myelinscheiden gequollen und teilweise zerfallen waren; eine Gewebsreaktion war hier kaum erkennbar und beschränkte sich auf einzelne Gitterzellen und Faserbildner. Auf diese Zone folgte eine zweite ebenfalls schmale, in deren Bereich das Myelin wesentlich stärker abgebaut war und welche Gitterzellen in beträchtlicher Zahl enthielt. Auf diese folgte die Hauptzone, in welcher der Myelinabbau voll im Gange war.

Ähnliche Bilder lieferten auch Spielmeyer-Schnitte: Am Rande der Herde war die Entmarkung nicht vollständig und es färbte sich das im Abbau begriffene Myelin blaßblau, mit allmählichem Untergang zum dunkelblau im gesunden Gewebe und grau im erkrankten. Durch Überfärben dieser Schnitte mit Scharlach war es möglich, den Abbau des Myelins za verfolgen.

Die Holzersche Methode zeigte überall in den geschädigten Arealen einen großen Faserreichtum sowie eine dichte perivasculäre Gliose. Die Achsenzylinder erwiesen sich in Bielschowsky-Schnitten als sehr stark gelichtet, Goldsublimat brachte hauptsächlich große Faserbildner zur Darstellung. Die Hortegafärbung ist nicht gelungen.

Weigert-Schnitte: Horizontalschnitte durch beide Hemisphären ergaben eine einseitige Erkrankung (Abb. 4). In der linken Hemisphäre war das Mark des Occipital, sowie fast des gesamten Temporallappens vernichtet. Die Störung griff außerdem auf das hintere Knie der inneren Kapsel, einen Teil des Thalamus und des Putamen über. Kleine Herde bestanden in der Inselrinde. Schließlich (im Bilde nicht sichtbar) war das ganze subthalamische Gebiet, die Brücke und die grauen Kerne am Boden des 4. Ventrikels mit Ausnahme der Oliven in Mitleidenschaft gezogen worden.

Die entmarkten Areale stellten einen nahezu zusammenhängenden Herd dar, welcher an einzelnen Stellen von schmalen Myelinstreifen durchzogen wurde. In den tieferen Lagen der Herde war die Entmarkung meistens eine vollständige oder doch nahezu vollständige, und nur in einzelnen Stellen waren feine Myelinringe oder Wellen erhalten. Diese Erscheinung war an der Peripherie wesentlich deutlicher ausgeprägt, hier waren zahlreiche Ringe, Wellen und auch kleine perivasculäre 


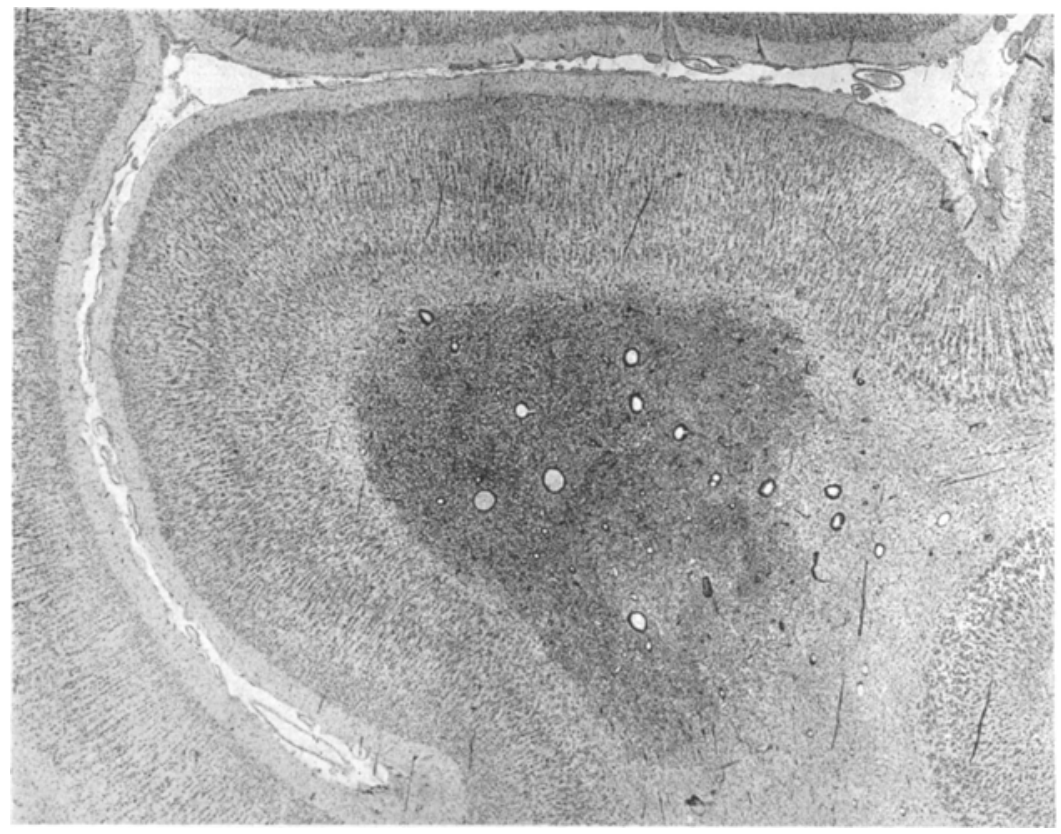

Abb. 2. Größerer Herd im Mark des linken Occipitallappens. Nisst-Färbung; 10mal. Mikrophotogramm.

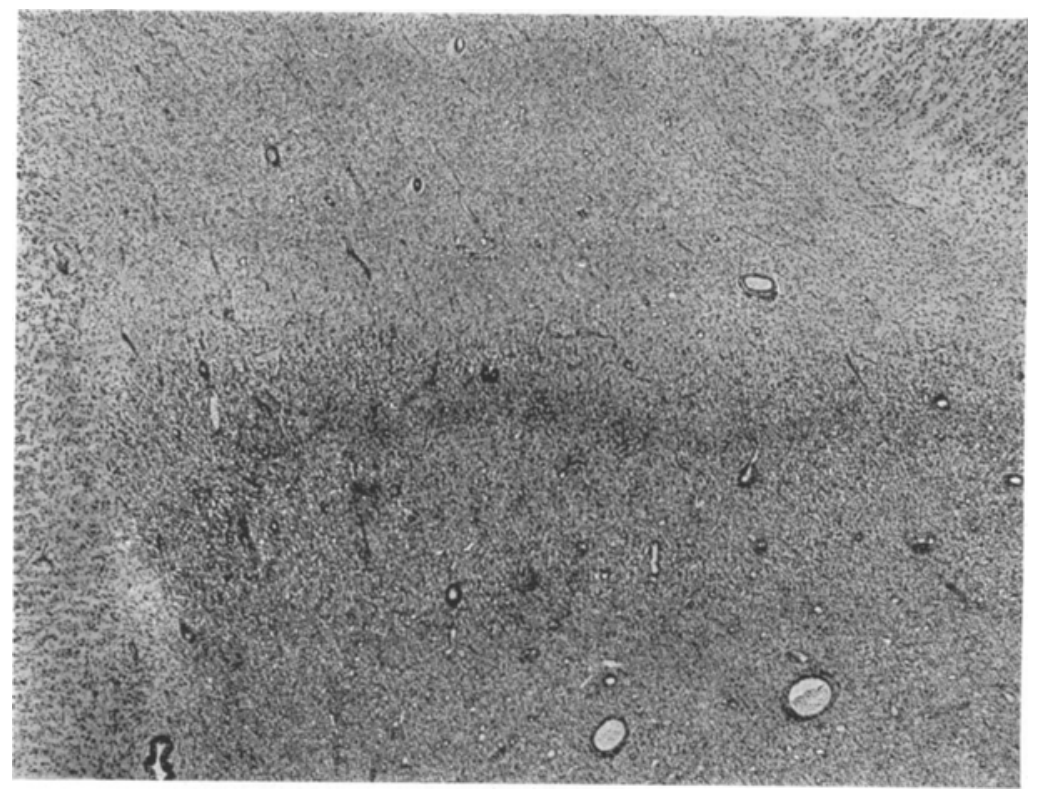

Abb. 3. Deutliche Wallbildung an einem größeren Herd des Occipitallappens. Nissl-Färbung: $10 \mathrm{mal}$. Mikrophotogramn. 
Markinseln nachweisbar (Abb. 4 und 5). Im Occipitallappen umgriff der Herd das Hinterhorn des Ventrikels, doch blieb das Ependym verschont.

In der Brücke war die Entmarkung eine weit fortgeschrittene, die Myelinringe waren hier nur undeutlich erkennbar; dagegen blieben in der Medulla feine, in regelmäßigen Abständen gelegene Marklamellen erhalten. Die Oliven und die Pyramiden

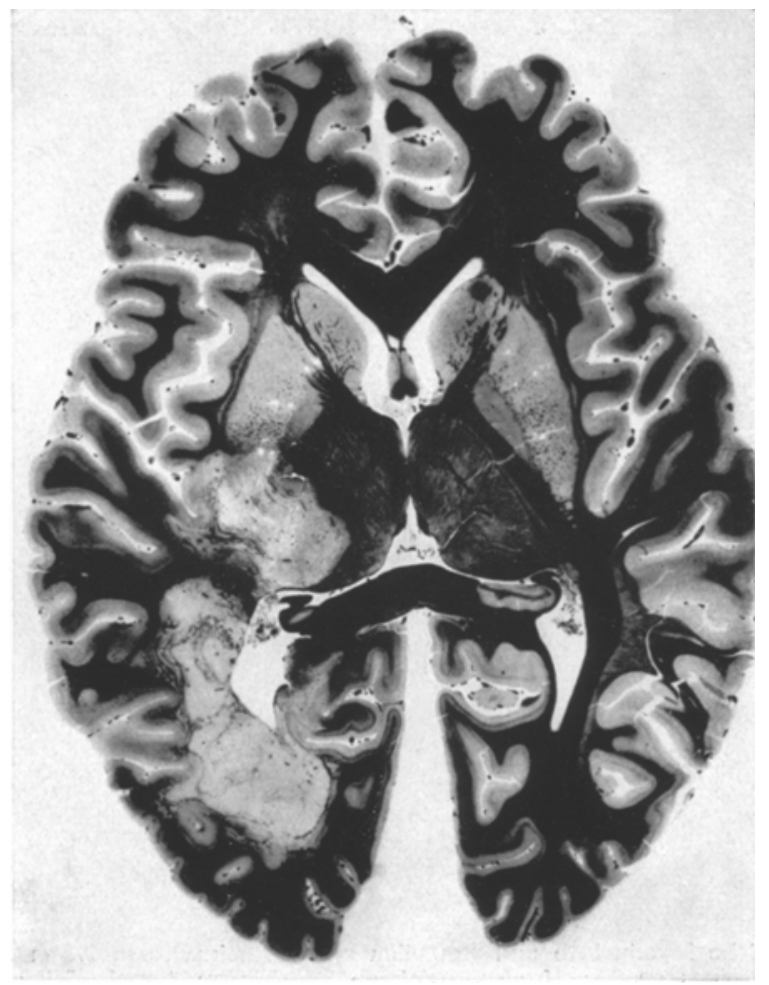

Abb. 4. Entmarkung des linken Occipitallappens und eines Teils der basalen Ganglien. An der Peripherie zahlreiche Myelinringe und Wellen. Weigert-Kultschitzky; ${ }^{2 / 3}$ nat. GröBe. Photographie.

blieben im wesentlichen verschont, während der ventrale Teil der Substantia reticulata alba zerstört war (Abb.6). Das Cerebellum und das Rückenmark waren intakt.

\section{Zusammenfassung.}

Histologisch handelt es sich um kleine perivasculäre Rindenherde, welche sehr oft zusammenfließen und dadurch große Abschnitte der weißen Substanz einschmelzen. Die Herde enthalten hauptsächlich Astrocyten, verschiedene Entwicklungsstufen der Hortegazellen sowie Lymphocyten. Die Faserproduktion ist sehr reichlich, Achsenzylinder sind schwer geschädigt. Der Lipoidabbau vollzieht sich in der üblichen Weise. 
Die Erkrankung ergreift die linke Hemisphäre, einen Teil der Stammganglien, den Gehirnstamm und das verlängerte Mark.

Die entmarkten Areale können in 2 Typen eingeteilt werden: 1. große Herde, welchen man bei diffuser Sklerose begegnet mit dem Unterschied, daß hier an der Peripherie zahlreiche Myelinwellen erhalten sind, und 2. kleinere runde oder ovale Herde, welche an multiple Sklerose erinnern.

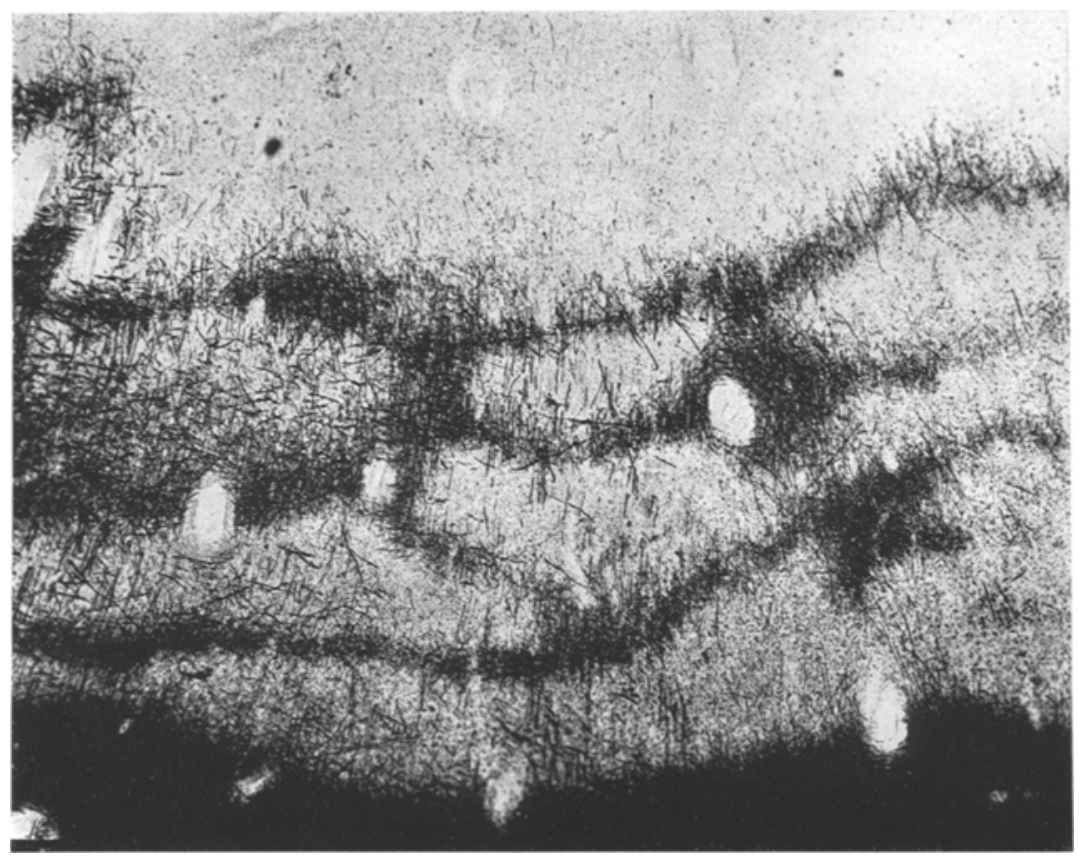

Abb. 5. Zahlreiche Myelinringe und Lamellen sowie vereinzelte perivasculäre Inseln an der Peripherie eines großen Herdes. Weigert-Kultschitzky: 10mal. Mikrophotogramm.

Klinische Besprechung. Von den 7 bis jetzt bekannt gewordenen Beobachtungen (die vorliegende mitgerechnet) betrafen 6 junge Menschen. Der jüngste Fall war ein 10jähriger Knabe (Patrassi), unser Kranker war 17 Jahre alt, derjenige Balós 23, der 1. Fall von HallervordenSpatz 24, die Kranke Marburgs 30 und nur die 2. Patientin von Hallervorden-Spatz steht mit 51 Jahren etwas abseits. 4 von den Kranken waren Männer, 3 waren Frauen. Der Krankheitsbeginn kann ein schleichender sein, wie es Patrassi beobachtet hat und mit Kopfschmerzen und Schwindelanfällen einsetzen, welche von Perioden vorübergehender Besserung unterbrochen werden. Umgekehrt schildert Marburg einen plötzlichen schweren Krankheitsbeginn mit Kopfschmerzen, Schlafsucht und Delirien. Die französischen Autoren (Barré) fanden Doppeltsehen, sowie Schwäche der Extremitäten. Batós Kranke klagte über 
Sprach- und Schreibstörungen, sowie Unsicherheit in den Händen. Auch der erste Fall von Hallervorden-Spatz hatte zunächst Schwierigkeiten beim Schreiben, sowie Parästhesien und Lähmung der rechten Hand. Bei unserem Kranken traten die ersten bedrohlichen Symptome im Anschluß an ein Kopftrauma in einer so auffälligen Weise auf, daß es gerechtfertigt erscheint, in diesem ein provozierendes Moment zu

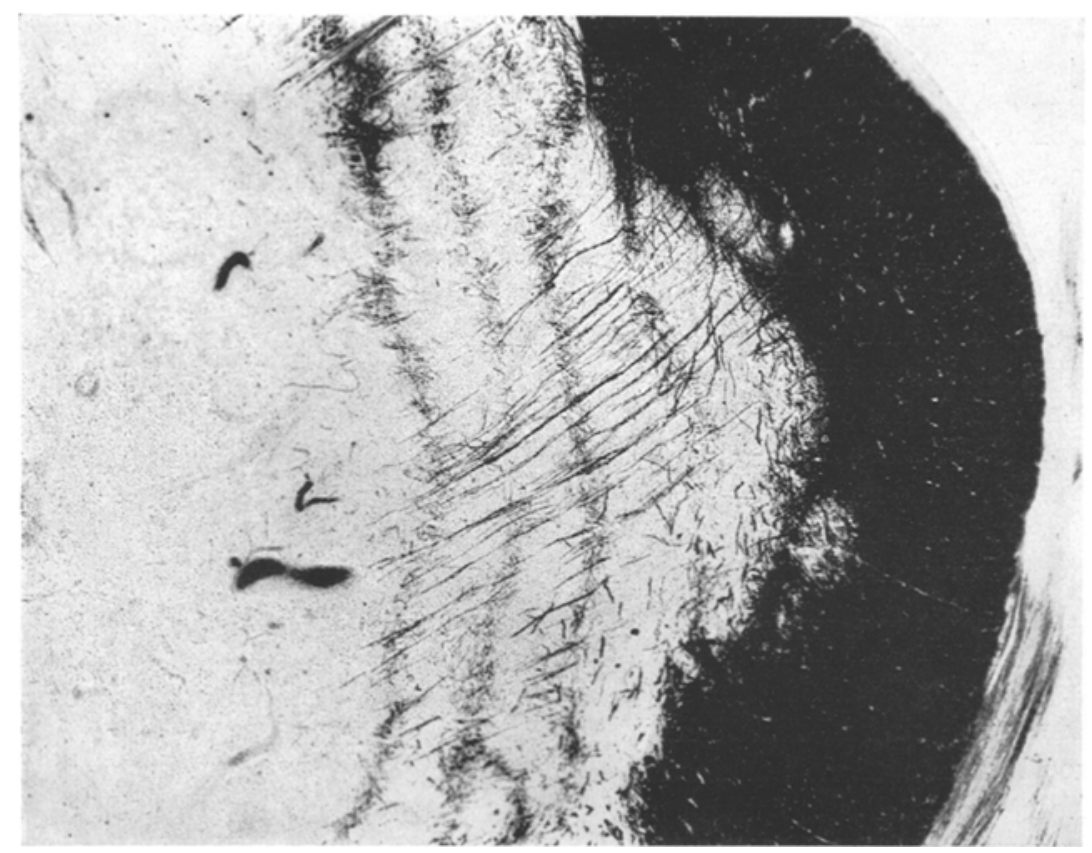

Abb. 6. Konzentrische Entmarkung im verlängerten Mark; mehrere in regelmäßigen Abständen liegende Lamellen sind erhalten. Weigert-Kultschitzky: 10mal. Mikrophotographie.

erblicken. Schwere Symptome schließen sich im Laufe von wenigenWochen an und gestalten das Bild immer bedrohlicher. Am akutesten war der Verlauf bei der Kranken Marburgs, welche soporös wurde und bald darauf starb. In der Mehrzahl der Fälle entwickelt sïch ein schweres Krankheitsbild, welches durch Lähmungen der Hirnnerven, Neuritis optici oder Stauungspapille, Sprach- und Gehstörungen, Lähmungen der Extremitäten, der Blase und des Darmes sowie durch Auftreten von pathologischen Reflexen ausgezeichnet ist.

In unserem Falle waren die vegetativen Störungen besonders ausgeprägt, was vielleicht auf die schweren. Veränderungen des Gehirnstammes zurückzuführen ist.

Die Krankheitsdauer ist großen Schwankungen unterworfen und variiert zwischen 4 Wochen (Marburg) und 2-3 Jahren (Barré); in den 
meisten Fällen betrug sie 4-16 Wochen. Die klinische Diagnose war in keinem Falle möglich; Laboratoriumsuntersuchungen erwiesen sich als wertlos.

Der Versuch einer Klassifizierung muß sich vor allem auf die Art der Entmarkung stützen. Die bis jetzt beschriebenen Fälle sind aber morphologisch nicht ganz einheitlich. Am reinsten ist die diskontinuierliche Entmarkung in den Beobachtungen von Hallervorden-Spatz sowie Baló ausgebildet; sehr ähnliche Befunde sind auch von Marburg sowie Barré und seinen Mitarbeitern erhoben worden. Die Entmarkung war in den Fällen von Hallervorden-Spatz und Baló so deutlich, daß die Ringe photographisch festgehalten werden konnten. Auch die französischen Autoren sprechen von einer moireartigen Zeichnung. Nach HallervordenSpatz legen sich ,,unregelmäßig gestaltete Lamellen von grauer Farbe als konzentrische Ringe oder Halbkugeln aneinander, in dem immer 2 graue Lamellen von einem Streifen unveränderter weißer Substanz geschieden werden. Das Bild erinnert an den Querschnitt eines Baumstumpfes, oder einen Achatschliff.“ Diese Schilderung ist auch für den Fall Bałós zutreffend. Dagegen haben wir bei der Zerlegung des Gehirnes nichts von einer diskontinuierlichen Entmarkung wahrgenommen, wir hatten den Eindruck eine diffuse Sklerose vor uns zu haben, und der Nachweis von Myelinringen im Schnitt kam uns gänzlich unerwartet. Auch bei einer nachträglichen Überprüfung der unbenutzten Blöcke konnten wir eine diskontinuierliche Entmarkung nicht wahrnehmen. Die Ursache liegt wohl in der Feinheit der noch erhaltenen Myelinlamellen. Die Ringe sind vielfach sehr klein, oft kaum größer als ein Stecknadelkopf, andere wiederum linsengroß, und nur relativ wenige sind größer. Aber gerade diese letzteren sind von sehr zarten Markstreifen gebildet. Auch liegen die meisten Ringe und Wellen an der Peripherie der großen Herde, was den Eindruck erweckt, daß die diskontinuierliche Entmarkung ihrem Ende nahe ist. Es muß aus dem Gesagten geschlossen werden, daß die Unsichtbarkeit der rhythmischen Entmarkung am ungefärbten Material ihre Anwesenheit keineswegs ausschließt.

Auch in der Ausbreitung der Erkrankung sind Varianten festgestellt worden; so waren im vorliegenden Falle die linke Hemisphäre, der Gehirnstamm, Brücke und verlängertes Mark befallen, während in den übrigen Fällen beide Hemisphären, wenn auch nicht immer symmetrisch, ergriffen waren.

Hallervorden-Spatz haben im Nissl-Präparat ein positives Bild zu dem negativen der Markschnitte feststellen können. Die entmarkten Streifen traten ihres Zellreichtums wegen als dunkler gefärbt hervor. Ein solches Verhalten haben wir nicht beobachtet, vielmehr erschienen die größeren Herde im Thioninpräparat als intensiv gefärbte Markareale, was dem Weigert-Schnitt im allgemeinen entsprach. 
Beim Vergleichen der bis jetzt in der Literatur niedergelegten Beobachtungen kann man sich des Eindruckes nicht erwehren, daß die konzentrische Sklerose eine Reihe von Abstufungen mit allmählichem Übergang zur diffusen Sklerose aufweist. Der vorliegende Fall ist in dieser Hinsicht besonders lehrreich, da das Mark der linken Hemisphäre 2 große, nahezu zusammengeflossene Herde enthält, welche mit denen der diffusen Sklerose weitgehend übereinstimmen. Trotzdem ist es aber nicht ratsam, Beobachtungen wie die hier besprochene mit der diffusen Sklerose zusammenzuwerfen, dazu sind die pathologisch-anatomischen Befunde in mancher Hinsicht viel zu abweichend. Solange die Ätiologie unbekannt bleibt wird man gut tun, dieselben in eine besondere Gruppe einzuordnen.

Histologisch sind sämtliche Beobachtungen im wesentlichen übereinstimmend, das Bild wird vom Myelinabbau beherrscht. Dementsprechend schildern sämtliche Autoren große Mengen von fettbeladenen Gitterzellen, Astrocyten und gliöse Riesenzellen. Holzer-Präparate ergeben große Mengen von Fasern; perivasculäre Infiltrate aus Lymphocyten bestehend gehören zur Regel. Ungewöhnlich ist dagegen das Auftreten eines zellreichen, peripheren Walles, wie Hallervorden-Spatz und wir ihn beobachtet haben.

Das Wesen der konzentrischen Entmarkung ist noch nicht ausreichend geklärt. Die Lösung ist in 2 verschiedenen Richtungen gesucht worden: 1. In histologischer, 2 . in kolloid-chemischer.

Es kann im vorliegenden Falle keinem Zweifel unterliegen, daß die Herdbildung stets perivasculär einsetzt und daß das Gefäßrohr von zahlreichen kleinen Entmarkungen ring- oder traubenförmig umgeben wird. Da dieser Vorgang im Gehirn dreidimensional verläuft und die Herde sich wie Ölflecke ausbreiten, müssen Myelinringe oder Wellen entstehen, so lange die einzelnen Herde nicht gänzlich zusammengeflossen sind. Wie die Fettpräparate beweisen, besteht in den Anfangsstadien der Myelinzerstörung so gut wie gar keine Gewebsreaktion, so daß man förmlich gezwungen ist, an Diffusion eines myelinauflösenden Stoffes durch das erkrankte Gefäßrohr zu denken, wie es Redlich schon vor Jahren getan hat. Hier knüpfen Hallervorden-Spatz ihre kolloid-chemischen Betrachtungen an. Spatz hat durch intravitale Farbstoffinjektionen nachgewiesen, daß das Eindringen des Farbstoffes im wesentlichen von seiner Dispersität abhängt, und Hallervorden erzielte rhythmische Niederschläge im Kaninchengehim durch Durchtränken mit Silbernitrat. Die Gewebsstrukturen spielen dabei keine besondere Rolle, so daß ähnliche Verhältnisse vorzuliegen scheinen wie bei der Entstehung von Liesegangschen Ringen. Die Autoren nehmen daher eine Diffusion als Ursache der Entmarkung an. So bestechend die Diffusionstheorie auch ist und so gut sie den histologischen Tatsachen gerecht zu werden scheint, darf nicht vergessen werden, daß diese Anschauung vorläufig rein hypothetischer Natur ist. Die Diffusionstheorie ist genau so wenig wie die 
Histologie imstande, etwas über die Natur der Schädlichkeit auszusagen. Bielschowsky hält es für sehr unwahrscheinlich, daß ein lebendes Virus eine so eigenartige Veränderung hervorrufen kann und in der Tat ist der Nachweis eines Erregers bis jetzt in keinem Falle gelungen; Färbungen nach Gramm sowie Silberimprägnierungen nach Steiner und Jahnel waren auch in diesem Falle negativ.

\section{Literaturverzeichnis.}

Baló: Encephalitis periax. concen. Areh. of Neur. 19, 242 (1928). - Barré, Morin, Draganesco et Reys: Encephalite periaxiale diffuse. Revue neur. 2, 541 (1926). - Bielschowsky u. Henneberg: Über familiäre diffuse Sklerose. J. Psychol. u. Neur. 36, 131 (1928). - Hallervorden-Spatz: Utber die konz. Sklerose und die physikalisch-chemischen Faktoren bei der Ausbreitung von Entmarkungsprozessen. Arch. f. Psychiatr. 98, 641 (1933). - Marburg: Die sog. akute multiple Sklerose. Jb. Psychiatr. 27, 213 (1906). - Patrassi: Diffuse Gehimentmärkungen und sog. Encephalitis periaxialis diffusa. Virchows Arch. 98, 281 (1931). - Redlich: Diskussionsbemerkungen. Verh. Ges. dtsch. Nervenärzte. Würzburg 1929. 